\title{
Mass and New Media: Review of Framing, Treatment and Sources in Reporting on Fukushima
}

\author{
Perko $\mathrm{T}^{1 *}$, Mays $\mathrm{C}^{2}$, Valuch $\mathrm{J}^{3}$ and Nagy $\mathrm{A}^{1}$ \\ ${ }^{1}$ Belgian Nuclear Research Institute, SCK·CEN, Belgium \\ ${ }^{2} S Y M L O G$, France
}

${ }^{3}$ Social Media Consultant, Czech Republic

\begin{abstract}
How was information about the Fukushima Daiichi nuclear disaster presented and transmitted in traditional and new media? How did these two major families interact in the post-Fukushima media dynamic? We reviewed 30 scientific publications or presentations dealing with mass media behaviour and its effects in the case of the March 2011 Fukushima accident. Data from Japan and also from other world regions were considered. Our structured review considered the framing and interpretation found in the media and the sources cited. A brief online survey also was conducted to complete our understanding of how European institutions applied social media in this crisis. Traditional media were found to provide context including frequent comparisons with the Chernobyl accident, however the expert technical vocabulary of radiation appeared incompletely translated. The number and range of cited sources grew in the days following the accident. Institutions used social media mainly as an expedient when traditional websites crashed under the weight of consultation. In contrast, a form of 'citizen journalism' emerged when social networks sped, decentralised and diversified information provision while offering platforms for direct citizen participation, expression and feedback. This dynamic offers opportunities for moving closer to a citizen-centred ideal of risk communication.
\end{abstract}

Keywords: Risk communication; Ionizing radiation; Mass media; New media; Fukushima; Survey

\section{Introduction}

Media studies take it as a commonplace that mass media are the most prominent information channel related to risk communication with the general public. Communication scholars point out that journalists have an active role to play in reporting about e.g. a risk event (or crisis) [1]. They represent, interpret, and construct it, and in this way they partly drive the related political and public salience of various issues $[2,3]$. Journalists do not only report about reality, they also influence it.

Risk communication in democratic societies indeed is an interactive exchange of information and opinions concerning risks and risk management among risk assessors, risk managers, consumers and other interested parties, usually referred to as stakeholders; this process of exchange relies notably on various means of mass communication [4]. Mass media, traditional (for instance newspapers, radio or TV) and new (for instance Internet, social websites, video sharing, micro blogging) may all be vectors through which risk information and evaluations are circulated at the level of the general public. In this article we use "risk communication" to refer loosely to formal and informal, intentional and empirical, directed and multidirectional processes of transfer and construction of risk-related content.

This article provides an analytical and critical review of how information related to the risks of ionising radiation is transmitted in the mass media (traditional - e.g. print, radio, television, and new Internet-based). In addition, a dynamic process between traditional and new media (social media) is analysed. The mass media are of interest for their role not only in distributing (educational) information related to ionizing risks, but also in presenting and clarifying different stakeholders' views and opinions important for democratic risk governance.

We review finding of 30 published scientific studies of the mass and social media treatment of the March 2011 Fukushima accident in
Japan, Europe, and North America. The analysis gives insight into how journalists understood and re-coded the information related to the Fukushima nuclear accident, and which sources provided journalists with information. Complementary data on the treatment in new and social media were obtained through a specific web-based questionnaire administered to a convenience sample in several European countries.

\section{Data Collection}

In order to identify how traditional mass media in Japan, North America and European countries reported about the Fukushima nuclear accident we performed a review of scientific articles on this topic. To constitute our sample of articles in October 2013 we searched the Web of Science, Google Scholar and Microsoft Bing literature databases using keywords "Fukushima" and "media". This research resulted in 73 hits on Web of knowledge. Fifteen relevant articles remained after removing double entries, and after evaluating the title and abstract. We applied the following criteria on the title and abstract selection: 1) the manuscript should be a research article in a scientific journal or a book, or be presented at a conference and 2) be about mass media related to the Fukushima accident. Fifteen further relevant studies were yielded by Google Scholar and Microsoft Bing. Thus 30 articles were selected for in-depth analysis. They analyzed media behaviour closely linked in time to the Fukushima accident or at anniversary dates. Some of the selected articles also brought in comparative aspects such as past media

*Corresponding author: Perko T, Belgian Nuclear Research Institute SCK·CEN, Belgium, Tel: 322655759/+3214332851; E-mail: tperko@SCKCEN.BE

Received March 24, 2015; Accepted March 30, 2015; Published April 05, 2015

Citation: Perko T, Mays C, Valuch J, Nagy A (2015) Mass and New Media: Review of Framing, Treatment and Sources in Reporting on Fukushima. J Mass Communicat Journalism 5: 252. doi:10.4172/2165-7912.1000252

Copyright: ( 2015 Perko T, et al. This is an open-access article distributed under the terms of the Creative Commons Attribution License, which permits unrestricted use, distribution, and reproduction in any medium, provided the original author and source are credited. 
behaviour related to historic accidents (like Chernobyl or Three Mile Island). In reviewing these publications, we refer loosely to "European" media when several different media from a range of individual European member states were studied (i.e., this term does not refer to pan-European or supra-national media).

For the analysis of new media we followed two approaches for data collection. We collected field data via an original online questionnaire sent to a convenience sample of involved institutions in some European countries; Austria, Belgium, Slovenia, Romania, France, Poland and Greece. The results should not be considered as representative; rather they indicate some aspects which could be followed up in future research. We also reviewed scientific articles that mainly discuss new media engaged during the communication of the Fukushima accident.

\section{Results}

\section{Traditional media}

Patterns of media coverage: Traditional media attention to ionizing radiation risks arising as a consequence of the Fukushima nuclear accident was intense and worldwide. TV broadcasters in the US (e.g. CBS, NBC, ABC) reported on the Fukushima accident regularly every night from the first day of the accident sequence on $11^{\text {th }}$ March, 2011 until the 18th of March 2011 [5]. In European media coverage, similar initial high media attention at the beginning of the accident is observed as well as a drop in media attention after a few weeks of the beginning of the accident. Cantone et al. observed in Italy, Belgium and Slovenia that media attention to the accident decreased over time, despite the fact that emergency management continued to remain a challenge at the nuclear power plant and was expected to have broad radiological, social and economic consequences [6]. The Fukushima coverage faded as other news topics took precedence. "The media are interested to report about the crisis events and not the processes or the continuous development of the accident consequences, for instance long releases and long environmental remediation works afterwards. They are more interested on the risk related to the immediate consequences than on the safety standards" [6]. The authors argued that communicators have to be prepared for the media (over)pressure at the beginning of the event and for the decrease of media attention in the later stage. This bears consequences especially for the process of environmental remediation or long term communication during risk assessment of nuclear installations [6].

In traditional media the Fukushima nuclear accident was often presented through the prism of the past nuclear accidents. A content analysis of 277 news articles found notable differences of overall traditional media reporting when comparing older articles written in response to past accidents and articles reporting on the Fukushima accident [7]. Kim and Bie found that U.S. news articles reporting on the Fukushima accident were more likely to provide quantitative and factual information than did articles on e.g. Chernobyl or Three Mile Island. Friedman concluded that "after the Fukushima nuclear accident, traditional media produced articles that put the ionizing radiation news into perspective, exploring the continuing scientific dispute over the effects of long-term exposure to low doses of radiation, the degrees of danger from radiation and how radioactive elements travel in the atmosphere, are deposited, and contaminate food chain" [5,7].

Perko did not find such perspective in Flemish newspapers reporting at the first anniversary of Fukushima accident. She identified a communication challenge related to the vocabulary used by experts or the media to describe radiological risks. Although experts are accustomed to communicating ionizing radiation risks in a technical language, often using quantitative units to present risks, mass media typically don't use these units in their reporting. Only about $12 \%$ of all articles published in analysed newspapers contained radiation units. The measurement unit most often used was the Becquerel (38\% among the articles reporting measurement units) and only $8 \%$ of the articles used qualitative comparison with familiar radiological exposures (such as X-rays) in order to present radiological risks [8].

Historical nuclear accidents were often used as a reference point by European mass media reporting about the Fukushima accident $[6,9]$. The word "Chernobyl" appeared in articles in various European media almost every day and comparisons were made repeatedly between the two accidents. This allowed Cantone et al. to infer that the European journalists presented the Fukushima disaster through the frame of the Chernobyl accident.

The content analysis by Katchanovski shows that both American and Canadian TV networks mostly presented the Fukushima nuclear accident as less severe than the Chernobyl nuclear accident. "When Fukushima was rated at the maximum 7 level on the International Nuclear and Radiological Event (IAEA) Scale, many news reports for the first time noted its similarity to Chernobyl" [10].

Katchanovski suggests that political or commercial alliances at the national level may (indirectly) influence media discourse. "The American and Canadian coverage of the Fukushima nuclear accident, which happened in the territory of an ally of the U.S. and Canada, differed significantly in most quantitative and qualitative aspects from the coverage of the Chernobyl nuclear disaster in Ukraine, which was not an ally" [10].

Similarly as observed in the U.S., newspapers in Europe at the outset of the accident focused their attention on multiple topics; yet, attention decreased with time and became limited to the future of nuclear energy and the safety and crisis management aspects in the country [6]. In newspapers in Italy, Germany, France, United Kingdom, Switzerland, Slovenia and Belgium, the nuclear accident was (ab)used in the media coverage as a frame for political discussions about the national future of nuclear energy, for instance a referendum on nuclear energy in Italy $[6,11]$. Prezelj et al. uncovered influence of a political/ideological discussion related to the accident and the future of nuclear energy in the European media as well: "In the states with a vivid public debate about nuclear energy, one of the strongest voices were the politicians, while in the states where nuclear energy is a silent topic, the research institutes had a stronger voice in communication about the accident." [12]

Public or private ownership of a medium influenced journalistic understanding and re-coding of the risk information related to the Fukushima accident. For instance, the NBC television network in the U.S. presented the accident in Japan as less severe than Chernobyl, "reports favourably contrasted the reactor design in Fukushima to that of Chernobyl, and they did not broadcast criticism of certain elements of the Fukushima reactor design by experts" [10]. The authors explained this anomaly by the effect of ownership of the NBC television network by General Electric, which designed the nuclear reactors in Fukushima. Convergence in the coverage outweighs political culture differences between the U.S. and Canada [10].

Information sources related to risk in the traditional media: In general, in traditional media coverage of ionizing radiation risks related to the Fukushima nuclear accident "the problem wasn't getting expert sources; it was vetting experts sources. Everyone with broadband 
had access to more expertise than a reporter could possibly read and absorb" concluded Sandman in a discussion related to the experts' role in explaining radiological risks in the U.S [13]. A research finding from Europe supports this statement. Prezelj et al. investigated which information sources actually reached the general public through the mass media during the event phase of the Fukushima nuclear accident in newspapers from various European countries [12]. The results show that, in the first 24 hours after the radiological emergency, only a limited range of information sources were cited, while subsequently the number of information sources increased significantly to include a broad spectrum of domestic sources, external and Japanese sources. The most quoted domestic sources in each studied country turned out to be opinion makers, the government and the nuclear safety authority. At the same time, the most quoted external sources were foreign governments, opinion makers and the IAEA, while the most quoted Japanese sources were the government, TEPCO and the inhabitants [12].

In general, for US and European newscasts, early reports about the accident relied heavily on amateur videos and getting reporters at the place was an additional issue for TV news broadcasters [14]. On the other hand, Cantone et al.'s research from Italy, Belgium and Slovenia shows that newspapers acted as an arena for discussion, voicing the opinions of a plurality of stakeholders with different values and interests. The media in these three countries were found to seek information both from the affected population onsite in Japan (they report personal stories) and from the nuclear emergency authorities. The authors conclude that "even if a zone is evacuated, there will be journalists willing to enter the zone regardless of risks in order to be able to eyewitness the consequences of a nuclear event" [9].

\section{New media reporting in the Fukushima nuclear accident}

According to the online Merriam-Webster dictionary social media are any "form of electronic communication (e.g. Web sites for social networking and micro blogging) through which users create online communities to share information, ideas, personal messages, and other content (e.g. videos)".

We performed an informal online survey among a convenience sample of institutions providing public information about ionizing radiation risks in seven European countries. The survey asked officials to describe the use of new media or social media by their institutions in the period following the Fukushima accident and for ongoing information on the same subject. The results of the survey are reported here in a purely indicative manner and are not claimed to be representative.

According to the reports gathered by our survey, social media (SM) were used only very occasionally by the institutions consulted. Among respondents whose institution did use SM, there is a consensus on a good impact and future potential: "Not all possible means of communication were used, indeed, despite they possess great potential nowadays.", or: "During the crisis, we used mostly Twitter to share the information available on our website (direct links to our contents). After the crisis, we started using YouTube and Daily Motion to share movies made by our organization about the accident: how did it happen, what are the consequences on environment and health. We learned that social media give important signals about people's interrogations and need to be monitored fully from the beginning of an accident. At least 1 person full time needed just to monitor what people are saying and asking."

Reasons cited for "no use" or "no importance" of SM communication were "lack of understanding of what social media communication means", "distrust", and "lack of skills and experience". These responders point to agreement within their institutions (management, employees...) as to the potential of social media, however this agreement remains cautious rather than giving rise to enthusiastic action.

The decision to use social media channels was not always driven by the understanding of their potential, but simply by the need to find alternative channels of communication to webpages that kept crashing after peaks of visits or did not prove to be an effective channel for interaction. "The use of the social media (Twitter, Facebook) during the Fukushima nuclear accident was a decision made due to the web page technical problems caused by the increased number of visitors. The experience was positive; since then the use of the social media is established for regular communication too. We currently work on a social media strategy and rules of engagement." Quevenco reports that the IAEA experienced a similar situation, where the trigger to use social media channels more extensively was the inability to keep up with email requests and questions received from citizens and the need to pin up updates somewhere where people can subscribe to them [15].

The use of new media to present information and new media challenges: More than 900 million people use Facebook and there are more than 100 million tweets daily [16]. Not only individuals, but also information sources for, instance nuclear industry, have embraced social media as an important communication channel for crisis communication. In the fifth month after the Fukushima nuclear accident Google returned 73,700,000 results for the keyword "Fukushima" and 22,400,000 results for the keywords "Fukushima and radiation". On Google News, which tracks news coverage, there were 201,000 results for "Fukushima" and 20,100 results for "Fukushima and radiation" [5].

$\mathrm{Ng}$ and Lean observe that "the potential of social media has expanded far beyond the initial function of social communication among a network of friends. It has become an increasingly important tool in risk communication to allow the dissemination of timely and accurate information to global citizens to make more informed choices regarding a particular crisis. The Fukushima nuclear crisis is an example where the potential of social media was not fully tapped" [17].

Friedman observed that "while journalists contributed much of the news about Fukushima, citizens actively participated in blogs and on Facebook, Twitter, and YouTube, exchanging views and directing others to important news articles or videos [5]. This situation gave rise to citizen journalism. One of the important characteristics of citizen journalism is bypassing some of the bureaucratic procedures faced by media organizations to publish news and make it available. The Internet also gave the traditional media many opportunities for better coverage, with more space for articles and the ability to publish interactive graphics and videos. Coverage in The New York Times, for example, included an abundance of background and explanatory information about the Fukushima accident and radiation releases in multiple formats and gave readers the opportunity to better understand technical information."

The report by the Independent Investigation Commission on the Fukushima Daiichi Nuclear Accident (2012) suggests that official communications channels should pay more attention to taking part in and responding to online citizen communication. Unlike conventional government communication such as speeches and news releases, websites and social media are interactive and allow for twoway exchange of information. They are a ready channel for citizens to voice their concerns [18]. Currie reaffirms the belief that "the explosion of social media everything from social networking websites, to blogs, 
to broadcast text messaging has changed the way in which anyone involved in risk communications must look at overall communication plans. Especially in times of emergency, social media can and should be employed to transmit critically important information immediately to as many people as possible"[19].

New media and information sources: Based on the expert opinion in Perko et al., scientists offered extensive online tutorials about nuclear plants and radiation and science journals such as Nature and Science posted articles on their online news pages following 11 March 2011 [20]. Many private individuals and groups with Internet and socialmedia connections presented their own news, their interpretations of news from traditional media or their points of view on blogs, Facebook, Twitter, and YouTube. Hundreds of Twitter conversations appeared under a variety of hashtags such as \#fukushima, \#nuclear, and \#meltdown with people keeping each other up to date on events and where to find articles to read or videos to watch.

During the early days of the accident, when the Tokyo Electric Power Company and the Japanese government held news briefings to provide minimal and somewhat optimistic information, their reports were quickly interpreted, supplemented, and contradicted online by scientists, government personnel, nuclear industry or anti-nuclear sources, and private individuals. The global nature of the accident also diluted gatekeeper opportunities, as journalists, organizations, and citizens not only from Japan but also from the United States, Southeast Asia, Europe, Russia, Canada, and elsewhere shared news and comments about the accident [20].

Reporting on the Fukushima accident opened a space for a number of voices - from ordinary citizens to experts. The following example collected by Tsang and Komyia demonstrates how the personalised social media communication of one person who became a trusted source can turn into a massive information distribution channel [21].

An interactive website that was created and run by a professor at Tokyo University saw a significant spike in followers. The site explains radiation and nuclear energy data in layman terms and allows people to comment via his account (@hayano) on Twitter, the social media micro-blog. On the 7th of March, before the nuclear accident, the website had only 2255 Twitter followers. But by the 14th of March, it gained more than ten times as many followers, to 23,122 ; and by the 21st of March, there was a further six-fold increase, to 151,757. Later on, another 'citizen messenger' set up a website aggregating Prof. Hayano's Twitter posts in one page. This further encouraged more people visit Prof. Hayano's site.

Another example of crowdsourcing of citizen expertise and knowledge was introduced by Abe [22]. It is the online community called Hakatte Geiger. Established by Gogo Labs, Inc. on June 17, 2011, Hakatte Geiger, whose Japanese slogan inquires, "Will you measure [the level of nuclear radiation] by using a Geiger counter?", the site allows non-Geiger counter users to request volunteer Geiger counter users to measure nuclear radiation for them. The resulting reports are circulated via Twitter.Abe adds: "What these Geiger-reading communities have in common is that they all use digital networks, including social media, to connect people to produce collectively generated knowledge [22]. It is suggested that social media have provided tremendous, if not unprecedented, opportunities for people to participate in generating knowledge regarding the level of nuclear radiation. In practice, our knowledge on radiation risks has been shaped through the interaction between the distributed/circulated power of social media and the concentrated power of mass media (and scientists' communities).
One should never overlook the meaning of uses of social media in the Fukushima nuclear crisis".

Tsang and Komiya suggest that these communication tools, set up and run by members of the public, became a primary source for ordinary citizens to seek out and understand what was commonly perceived to be obscure technical information on radiation and nuclear fallout [21]. These tools are also self-policing, in that inaccurate information is often spotted and corrected by fellow users.

Tsang and Komiya also observed, that "while worries about the potential danger of radiation had inevitably driven citizens' hunger for news and information, the proliferation of online activity was spurred on by their lack of trust in official information put out by the government [21]. An opinion poll conducted by the Nomura Institute on 19 and 20 March, after the earthquake in Japan, $28.9 \%$ of respondents believed government information had become less trustworthy, while only $9 \%$ believed social media had become less trustworthy. In comparison, $7.8 \%$ of the respondents thought they trusted government information more, while $13.4 \%$ trusted social media more."

\section{Dynamics between traditional and new media}

Nowadays people can be selective about news they get and they can be actively involved in news production since they provide feedback about it. New media interfere with the traditional media, but it has not obviated the need for journalists on the ground. Sandman reports that "there was a greater flow of content from Fukushima reporters to online experts than from online experts to Fukushima reporters" [13]. Utz et al. demonstrated that journalists are credible information gatekeepers and traditional media are still recognised as more credible information source than new media [16]. However, the interaction of traditional journalism and "citizen journalism" nowadays makes communication a complex process.

Many traditional media (newspapers, TV broadcasters, radio) worldwide including BBC, The Wall Street Journal, The Washington Post, National Public Radio started to use extended information related to the Fukushima nuclear accident and its consequences available on the internet. This way they were giving more space and air-time for longer news and more in-depth reporting, along with complementary graphics, multimedia and links to updates from other web and social media [5]. Readers, viewers and the public in general "commented the news online, giving feedback to journalist and enabling dialogues that included opinions, answers to questions, and suggestions for other websites to view" [5].

Traditional media explanations were often borrowed by online experts' explanations [13]. Expert-volunteers had on line a dialogue with citizens and with other experts having different opinions, offering a rich information source for traditional media.

The authors of the report Perko et al. believe, that by this way of interaction between traditional and new media the radiation coverage at the Fukushima nuclear accident has been improved comparing to past major nuclear accidents [20]. The ionizing radiation communication has been more extensive and more emphasised on explanations and background information in general. The new media and graphical capabilities enabled traditional newscasts to visualise the information.

\section{Conclusions}

Against a background of theoretical and practical knowledge on the place of mass media in risk communication, the present article has reviewed 30 scientific publications dealing with mass media behaviour 
Citation: Perko T, Mays C, Valuch J, Nagy A (2015) Mass and New Media: Review of Framing, Treatment and Sources in Reporting on Fukushima. J Mass Communicat Journalism 5: 252. doi:10.4172/2165-7912.1000252

and its effects in the case of the Fukushima Daiichi disaster. Data from Japan and also from other world regions were considered in the publications reviewed.

The analysis of this literature enabled us to characterise the presentation and transmission of information post Fukushima in the traditional media while identifying the principal sources cited. The same literature lent itself to an analysis of the use of new media, and moreover, of the interactions between these two major families in the post-Fukushima media dynamic. Furthermore, a brief survey completed the vision of how the new (social) media were applied.

The overall image that emerges from these analyses is one of a fast-evolving context. It is a commonplace today that societal communication about risks has become more complex, extensive and multi-directional, and that nuclear accidents like Three Mile Island and Chernobyl, as well as other trans-border risk events, have largely contributed to this societal movement. However, new media appear to reinforce this movement, as they speed, decentralise and diversify information provision while offering platforms for direct citizen participation, expression and feedback. The growing presence of the new media and their interaction with the traditional media result in potentially greater challenges for institutions whose mission includes communication with the public about ionizing radiation risks in particular. At the same time, this dynamic offers opportunities for moving closer to a citizen-centred ideal of risk communication.

\section{Acknowledgement}

The article is a result of the Project EAGLE, funded under grant agreement $n^{\circ}$ 604521 by the European Commission Seventh Euratom Framework Programme for Nuclear Research \&Training Activities.

\section{References}

1. Franklin B, Hamer M, Kinsey M, Richardson EJ (2005) Key Concepts in Journalism Studies. SAGE, London.

2. Rupar V (2007) Newspapers' production of common sense; The 'greenie madness' or why should we read editorials? Journalism 8: 591-610

3. Vliegenthart R, Walgrave S (2008) The Contingency of Intermedia AgendaSetting. A Longitudinal Study in Belgium. Journalism and Mass Communication Quarterly 85: 860-877.

4. Renn O (2008) Risk Governance; Coping with Uncertainty in a Complex World Earthscan, London.

5. Friedman SM (2011) Three Mile Island, Chernobyl, and Fukushima: An Analysis of Traditional and New Media Coverage of Nuclear Accidents and Radiation. Bulletin of the Atomic Scientists 67: 55-65.
6. Cantone M, Perko T, Turcanu C, Prezelj I, Travola F (2012) The Fukushima Accident: Content Analysis of the Media in Three European Countries. AIRPAtti del XXXV Congresso Nazionale di Radioprotezione Radiation Protection Society, Venezia, Italy.

7. Kim J, Bie BJ (2013) A dangerous neighbor: The news frames of the radiation effects from the Fukushima nuclear accident. Risk Management 15: 180-198.

8. Perko T (2014) Radiation risk perception: a discrepancy between the experts and the general population. Journal of Environmental Radioactivity 133: 86-91

9. Cantone M, Perko T, Turcanu C, Prezelj I, Tavola F (2012) Content analysis of the media reporting on the Fukushima nuclear accident in three European countries. In 13th International Congress of the International Radiation Protection Association, Glasgow, Scotland.

10. Katchanovski I (2012) Fukushima vs. Chernobyl: Coverage of the Nuclea Disasters by American and Canadian Media. In Annual Meeting of the American Political Science. New Orleans.

11. Kepplinger HM, Lemke R (2012) Die Reaktorkatastrophe bei Fukushima in Presse und Fernsehen in Deutschland, Schweiz, Frankreich und England. In Jahrestagung. Hamburg: Die Strahlenschutzkommission.

12. Prezelj I, Tanja P, Catrinel T, Marie C, Cantone M (2012) Nuclear Emergency Communication: One Emergency, Many Voices. Nuclear Energy for New Europe, Ljubljana, Slovenia.

13. Sandman PM (2013) Media coverage of Three Mile Island versus Fukushima: Getting experts versus vetting experts.

14. Goodman T (2011) Japan disaster shows U.S. journalists unprepared. San Francisco Chronicle.

15. Quevenco R (2013) Social Media in Crisis Communication: The IAEA's Experience During the Fukushima Accident. IAEA 2011.

16. Utz S, Schultz F, Glocka S (2013) Crisis Communication online: How medium, crisis type and emotions affected public reactions in the Fukushima Daiich nuclear disaster. Public Relations Review 39: 40-46.

17. Ng KH, Lean ML (2012) The Fukushima Nuclear Crisis Reemphasizes the Need for Improved Risk Communication and Better Use of Social Media. Health Physics 103: 307-310.

18. IAEA ( 2011) Final Report of the International Mission on Remediation of Large Contaminated Areas Off-site the Fukushima Dai-ichi NPP, Japan: IAEA.

19. Currie D (2009) Exert Round Table on Social Media and Risk Communication During Times of Crisis: Strategic Challenges and Opportunities.

20. Perko T, Valuch J, Nagy A, Lammers P, Mays C (2013) Overview of Mass and New Media Treatment of lonizing Radiation Topics: The Case of Fukushima. Report. EAGLE Project Deliverable 2.1.

21. Tsang M, Masato K (2013) Lessons from Fukushima: Do not ignore citizen media.

22. Abe $Y$ (2013) Analyzing the Fukushima Nuclear Crisis through Uses of Socia Media: A Short Essay on a Work in Progress. 